\title{
¿Qué hacen los directores de centros escolares? Las prácticas de dirección en España a partir de los estudios internacionales PISA y TALIS
}

\section{What do school principals do? Leadership practices in Spain drawn from PISA and TALIS international studies}

\author{
David IZQUIERDO GÓMEZ
}

IES Julio Verne, Leganés, Madrid

Recibido: Diciembre 2014

Evaluado: Febrero 2015

Aceptado: Marzo 2015

\section{Resumen}

El papel del director es clave en los centros escolares. Sus acciones repercuten en la eficacia de los centros, y de manera indirecta en los resultados académicos de los alumnos. Numerosos estudios describen las diferentes actividades que desempeñan los directores en su práctica diaria, agrupándolas en dimensiones, no existiendo unanimidad en torno a las prácticas realizadas ni en las dimensiones en las que se agrupan. En España, la nueva ley educativa, LOMCE, hace hincapié en la importancia de mirar a lo que dice la investigación internacional en el ámbito de la educación para sustentar la necesidad de cambios y justificar los que se realicen, y desarrolla una lista extendida de las competencias propias del director. Este estudio es una síntesis de investigación narrativa cuya unidad de análisis son los resultados de los informes internacionales PISA (Programme for International Student Assessment) y TALIS (Teaching and Learning International Study) y los datos que aportan sobre la dirección y sus prácticas, a partir de los que se infieren unas conclusiones que se comparan con las competencias de dirección enunciadas en la LOMCE. El análisis comparativo de las prácticas de dirección presentadas describe un panorama aún heterogéneo y desigual, no existiendo coincidencia en la delimitación de las dimensiones de dirección, ni en el número de prácticas que las componen. Dicho panorama apunta a la necesidad de seguir investigando empíricamente para obtener con precisión un catálogo homogéneo de acciones desempeñadas por los directores que abra la puerta al desarrollo de la función directiva, y a la mejora de la efectividad y el rendimiento de los centros, tal y como se plantea a nivel internacional, y es objetivo en la nueva ley educativa.

Palabras clave: directores, estilos de liderazgo, política educativa, PISA, TALIS. 


\begin{abstract}
Principals play a key role in schools. Their actions affect the efficacy of schools and indirectly on the students' academic performance. Numerous studies describe the different activities that principals perform in their daily practice, grouping them in dimensions, not existing unanimity neither around the practices carried out nor the dimensions. In Spain, the new educational law, LOMCE, emphasizes the importance of looking into what international research says regarding the field of education to support the need for change and justify the ones that are being made, and develops an extended list of competences of the principal. This study is a synthesis of a narrative research with the following analysis unit: the results of the PISA (Programme for International Student Assessment) and TALIS (Teaching and Learning International Study) international reports, and the data provided on school leadership and their practices, from which conclusions are inferred, comparing them with the competences stated in the LOMCE. The comparative analysis of the leadership practices presented depicts a still uneven and heterogeneous scenario, not existing agreement neither for the setting of boundaries for the leadership dimensions, nor for the number of practices that constitute them. Such a scenario points towards the need for further empirical research, in order to accurately obtain a homogeneous catalogue of the actions carried out by principal, that may open the door to the development of the role of principal, and to the improvement of effectiveness and performance of schools, as it is suggested internationally, and it is an objective of the new educational law.
\end{abstract}

Keywords: principals, leadership styles, educational policy, PISA, TALIS.

\title{
La dirección de centros y sus prácticas: un escenario desigual
}

El liderazgo es un término que elude una clara definición (Yukl, 2002). Además, en el ámbito educativo, surge la dificultad de encontrar un equilibrio entre los elementos de liderazgo - cambio- y dirección - administración - a la hora de operacionalizar su definición (Cuban, 1988).

A pesar de la diversidad de planteamientos conceptuales a partir de los cuales desarrollar una fundamentación teórica homogénea que permita crear una definición clara, ha quedado sobradamente probado desde la literatura internacional la importancia del liderazgo escolar. Fullan (2002) llegó a decir que "los líderes escolares efectivos son clave para una reforma educativa sostenible y a gran escala" (p.15). Además, el informe McKinsey (2007) demuestra que los países con mayor rendimiento educativo desarrollan políticas relacionadas con el liderazgo educativo para estructurar las funciones, deberes e incentivos de los directores a fin de desarrollar líderes, sus capacidades y centrarlos en lo instructivo. Dicho informe hace referencia a los sistemas educativos de Singapur, Boston o Chicago como ejemplos de esas políticas.

De manera recurrente se ha dicho en diferentes investigaciones que el liderazgo escolar es segundo respecto a la instrucción en el aula como elemento que influye en el logro académico (p.e., Hallinger \& Heck, 1998; Mulford, 2003; Waters et al, 2005; Leithwood et al, 2006; Ingvarson et al., 2006; Seashore et al, 2010). Dichas investigaciones sobre el impacto del liderazgo de los directores en el aprendizaje de los alumnos han encontrado que su influencia se produce de manera indirecta, al no estar en contacto regular con los alumnos a diferencia de los profesores. Las áreas donde se encontró que el liderazgo escolar marca la diferencia fueron en las estructuras y 
procesos organizativas a nivel de escuela, donde producen efectos en el aula que inciden en el aprendizaje de los alumnos. Algunas de las prácticas más productivas de liderazgo mencionadas apuntan a: construir una misión, construir capacidad entre los profesores, y crear unas estructuras organizativas efectivas, aunque parece que no existe unanimidad en este respecto en los diversos estudios. Hasta tal extremo es así que en un estudio realizado por Grissom y Loeb (2011), se ha probado de manera positiva la relación de las prácticas organizativas con el rendimiento académico, tradicionalmente asociadas con el liderazgo burocrático y transaccional. De igual manera, la implementación de dichas prácticas se sigue realizando de manera tradicional por el director o por miembros de su equipo, lejos de las propuestas innovadoras de distribución de las tareas entre miembros de la comunidad educativa (Eurydice, 2013).

Leithwood, Harris y Hopkins (2008) llegan a plantear que todos los directores se basan en un mismo repertorio de prácticas, con independencia del entorno de trabajo. Por ello, es fundamental delimitar el conjunto de prácticas que son más efectivas. Uno de los obstáculos para lograr esto es la dificultad de encontrar datos sobre los que basar dicho análisis, al haberse probado los efectos como indirectos (Hallinger \& Heck, 1998; Witziers et al., 2003). Además, la definición de las prácticas realizadas en diferentes estudios no es clara, al listar diferentes áreas de responsabilidad y prácticas pertenecientes a cada una de ellas.

Desde el apartado VI del preámbulo de la Ley Orgánica para la mejora de la calidad educativa (LOMCE, 2013), se hace insistencia en que dos de los principios fundamentales sobre los que pivota la reciente reforma educativa son el aumento de la autonomía y el refuerzo de la capacidad de gestión de los centros mediante la rendición de cuentas. Ya la anterior Ley Orgánica de educación (LOE, 2006), en su preámbulo, incidía también en la importancia de la autonomía de los centros, y planteaba el establecimiento de mecanismos de evaluación y rendición de cuentas.

A los directores, como representantes de la Administración educativa en el centro y responsables del proyecto educativo, se les confiere la oportunidad de ejercer un mayor liderazgo pedagógico y de gestión - ampliando el abanico de competencias o prácticas que se les atribuye, tal y como está descrito en el artículo 132 de la LOMCE.

Así, frente a la diversidad de propuestas que intentan definir las prácticas de los directores y su liderazgo en los centros, este artículo pretende no partir de las conclusiones dadas por otros autores a la hora de analizar resultados. Este es el caso, por una parte, de las revisiones teóricas que se centran en las explicaciones teóricas comúnmente utilizadas para un campo en particular, que intentan explicar fenómenos usando alternativas teóricas, o integrar perspectivas teóricas múltiples; o el de las revisiones de estudios sobre prácticas típicas para un campo en particular. Por el contrario, se pretende tratar directamente con los resultados de las investigaciones (Card, 2012).

Para llevarlo a cabo, este artículo opta por la síntesis de investigación narrativa como método, cuya unidad de análisis son los resultados de los informes internacionales PISA (Programme for International Student Assessment) y TALIS 
(Teaching and Learning International Study) y los datos que aportan sobre las prácticas de dirección, a partir de los que se infieren conclusiones, y su posterior comparación con las competencias de dirección presentadas en la LOMCE. De esta manera, se refrenda la apuesta hecha por las administraciones educativas españolas para promover un cambio en base a la evidencia empírica. Se trata de alejarse de las tendencias de la literatura sobre liderazgo escolar - que no se han centrado tradicionalmente en las prácticas de los directores, sino más bien en las creencias, habilidades, conocimientos que los directores necesitan para ser efectivos en el trabajo (Leithwood et al, 2006) para mirar a lo que los estudios internacionales reflejan como prácticas habituales de los directores. Posteriormente, al contrastar los resultados de dicho análisis con lo que la nueva ley educativa presenta como el catálogo de las competencias propias de los directores españoles, se obtiene un panorama completo del estado de las prácticas de dirección.

Tal y como indica la LOMCE, el objetivo de este artículo es, por tanto, contribuir a la propuesta hecha por la ley de mejorar el rendimiento de los centros a través de una descripción de las prácticas de dirección.

Para lograrlo, en el siguiente apartado se describen a nivel general las prácticas de dirección encontradas en el informe internacional PISA, relacionándolas con los datos concretos para los directores españoles. De igual manera, se describe a nivel general las prácticas halladas en el informe internacional TALIS, realizando la misma comparativa con los datos aportados para los directores españoles. Posteriormente, se presentan los resultados de la comparación de dichas prácticas con las competencias de dirección de la ley educativa española. Finalmente, se presentan las conclusiones, que apuntan a futuras líneas de investigación.

\section{Los informes internacionales y la dirección de centros}

Se indica en la introducción de la LOMCE cómo los cambios propuestos en el sistema educativo están basados en evidencias. La reforma pretende hacer frente a los principales problemas detectados en el sistema educativo español sobre los fundamentos proporcionados por los resultados objetivos reflejados en los informes y evaluaciones periódicas de los organismos europeos e internacionales. Por ello, a lo largo de dicho apartado $\mathrm{V}$ del preámbulo, hace referencia a datos concretos procedentes de diferentes instituciones internacionales para justificar la necesidad de reformar aspectos del sistema educativo que se prueban no eficientes. En esta sección se pretende hacer una revisión de los informes PISA y TALIS, para recoger lo que dicen sobre la dirección de los centros, la figura del director y su práctica.

\section{La dirección en PISA}

La Organización para la Cooperación y el Desarrollo Económicos (OCDE) realiza de manera trianual desde el año 2000, mediante el Programa para la Evaluación Internacional de Alumnos (PISA), una evaluación de lo que los jóvenes de 15 años de diferentes países del mundo saben y son capaces de hacer en tres competencias 
consideradas troncales: matemáticas, lectura y ciencias. PISA elabora tendencias longitudinales para mostrar la evolución de diversos aspectos de los sistemas educativos en un plano comparativo internacional. Este artículo toma como referencia el estudio PISA del año 2012.

PISA 2012: Informe Español (INEE, 2013), en su volumen I apunta a las características de los centros educativos como uno de los factores que influyen en el rendimiento del alumnado: procesos internos del centro como el grado de autonomía, y la eficiencia en la gestión de los procesos organizativos y educativos entre otros. De la variabilidad total de los resultados académicos en el conjunto de los países de la OCDE, el $36.8 \%$ se debe a las características de los centros, siendo el caso de España del $17.1 \%$, que comparado con el porcentaje de la varianza encontrada en Finlandia, el $6.3 \%$, presenta al sistema educativo español como notablemente equitativo. Sin embargo, sigue siendo un porcentaje considerable los resultados académicos de los alumnos que se ven influenciados por las características de los centros. Puesto que la eficiencia en la gestión de los procesos organizativos y educativos de los centros es responsabilidad directa de las acciones de los directores, la diversidad de las prácticas de dirección apunta como causa a ese porcentaje de variabilidad. Por ello, se plantea necesario hacer una revisión más detallada de dichas prácticas, para localizar aquellas que incidan en el rendimiento académico. Este artículo apunta a esa línea de análisis.

La autonomía de los centros escolares, especialmente en lo relacionado con los contenidos, es mucho menor en España que en la media de países de la OCDE - 58\% frente al $82 \%$.

En cuanto a los mecanismos de rendición de cuentas, el informe nota una tendencia clara hacia el uso de resultados académicos en evaluaciones externas y estandarizadas, para comparar los centros educativos con la media nacional o regional de la OCDE. En la OCDE, el $45 \%$ de alumnos asisten a centros cuyos resultados académicos son hechos públicos, mientras que en España es solo del 13\%. Indica que la autonomía en la gestión de sus recursos basado en una rendición de cuentas aumenta el rendimiento académico, aunque dichos datos hay que tratarlos con cautela.

Finalmente, el informe español aporta un dato relevante: la poca variación de los resultados apunta a una relativa estabilidad del sistema educativo español desde el primer informe PISA del año 2000, lo que a su vez implica un estancamiento del sistema educativo. A esto alude la LOMCE cuando plantea la necesidad de realizar una serie de cambios basados en la investigación, y en lo que aquí se refiere, respecto a la dirección de centros.

What makes a school successful? (OCDE, 2013), volumen 4 del informe internacional del estudio PISA 2012, trata aspectos relacionados con la dirección de centros.

Empieza indicando que los centros con mayor autonomía para el diseño del currículo y la evaluación tienden a rendir mejor que aquellos con menor autonomía, cuando son parte de sistemas educativos con mayor rendición de cuentas. La razón expuesta para una mayor devolución de autonomía a los centros desde los años 80 se basa en la premisa de que los centros son mejores conocedores de sus propias 
necesidades y de las maneras más eficaces de adjudicar recursos y de diseñar el currículo para dar respuesta a las necesidades de sus alumnos - aspecto en el que la LOMCE incide de manera manifiesta.

El informe describe prácticas realizadas por los directores sobre las que tienen influencia directa, agrupadas en índices, y relacionadas con el impacto que tienen en el rendimiento de los alumnos. La información se obtiene de las respuestas de los propios directores y estudiantes a los cuestionarios de contexto que PISA realiza.

Entre la diversidad de tareas a desempeñar dentro de las diferentes dimensiones de la función de director, el informe describe que dan forma al desarrollo profesional de los profesores, definen los objetivos educativos del centro, aseguran que la práctica instructiva se encamina a lograr esos objetivos fijados, sugieren modificaciones al proceso de enseñanza, y ayudan a solucionar los problemas que surgen en el aula y entre la plantilla. Además, no solo lideran aspectos burocráticos, sino que son líderes instructivos.

Señala que España está a una distancia considerable por debajo de la media de la OCDE en el índice de responsabilidad escolar para la asignación de recursos (que trata prácticas de selección o despido de profesores, fijación de salarios y sus aumentos, y presupuesto escolar), con -0.42 y -0.05 respectivamente, indicando que la autonomía de los directores al tomar decisiones en estos aspectos es considerablemente menor, y que las autoridades educativas nacionales o regionales por separado, o bien compartiendo la autoridad, toman esas decisiones y las transmiten a los centros.

En cuanto al índice de responsabilidad escolar para el currículo y la evaluación (que trata prácticas de establecimiento de políticas de evaluación de alumnos, selección de libros de texto, determinación de los contenidos de los cursos, o decidir qué cursos se ofertan), España se encuentra de nuevo por debajo de la media de la OCDE, -0.47 y -0.04 respectivamente, indicando que la autonomía de los directores de los centros a la hora de tomar decisiones en estos aspectos es considerablemente menor (a excepción de la selección de libros de texto, donde se encuentra muy por encima).

Sobre la dimensión de distribución del liderazgo en los centros y las acciones que un director realiza para llevar a cabo dicha dimensión, PISA ha diseñado el índice de dirección escolar: participación del profesorado (que trata prácticas relacionadas con dar oportunidad a la plantilla para tomar decisiones, implicar a los profesores en una cultura de mejora del centro, y pedir a los profesores que participen en la revisión de prácticas de dirección) con objeto de determinar el grado de consecución del mismo, en el que España se encuentra ligeramente por encima de la media de la OCDE, 0 y -0.02 respectivamente.

El índice de dirección escolar: delimitar y comunicar los objetivos del centro y el desarrollo curricular (que trata prácticas como el uso de los resultados de rendimiento de los alumnos para desarrollar los objetivos educativos del centro, asegurarse que el trabajo de los profesores y sus prácticas de desarrollo profesional están en sintonía con los objetivos educativos fijados, y el debate sobre los objetivos académicos con los profesores en las reuniones del claustro), sitúa a España claramente por debajo de la media de la OCDE en esta dimensión, -0.30 y 0.01 respectivamente, planteando que 
dichas prácticas no son lo habituales o suficientemente eficaces en los directores españoles como lo son en las de sus homólogos a nivel internacional.

El índice de dirección escolar: liderazgo instructivo (que trata prácticas como la promoción de prácticas de enseñanza basadas en la investigación educativa reciente, la alabanza a profesores cuyos alumnos participan de manera activa en el aprendizaje, y el llamar la atención de los profesores sobre la importancia del desarrollo de las capacidades críticas y sociales de los alumnos), sitúa a España significativamente por debajo de la media de la OCDE en esta dimensión de dirección, -0.46 y 0 respectivamente, planteando que dichas prácticas tampoco son lo habituales o suficientemente eficaces en los directores españoles como lo son en las de sus homólogos a nivel internacional.

El índice de dirección escolar: promover la mejora en la instrucción y el desarrollo profesional (que trata prácticas como la toma de iniciativa para tratar asuntos cuando un profesor tiene problemas con su clase, prestar atención al comportamiento disruptivo en las aulas, y el plantear la solución de problemas junto al profesor cuando tiene algún problema en la clase), sitúa a España ligeramente por encima de la media de la OCDE, 0.16 y -0.02 respectivamente, planteando que dichas prácticas se realizan de manera más habitual y eficaz por los directores españoles en relación a sus homólogos a nivel internacional.

Si se mira a nivel sistémico, la relación entre autonomía y logro académico sigue existiendo, pero es mayor en aquellos sistemas donde se publican abiertamente los resultados de evaluaciones externas.

Según el informe, las evaluaciones estandarizadas externas sirven a los alumnos para incentivarles en el aprendizaje; a los profesores para comparar el rendimiento de sus alumnos con otros entornos y adaptar su pedagogía; y a los centros para determinar cómo se distribuyen los recursos y tomar medidas de intervención, e informar a la toma de decisiones políticas para trabajar encaminados a logros educativos establecidos de manera centralizada. Para evitar el impacto negativo de "enseñar para los exámenes", se plantea expandir el concepto de rendición de cuentas a los centros, profesores y directores, con la intención de ser utilizado con fines formativos.

El grado en el que los centros recogen información de sus alumnos sobre diversos aspectos relacionados con el funcionamiento del centro y lo hacen público muestra un mayor nivel de equidad en los sistemas, obteniendo un mayor rendimiento académico. El porcentaje de alumnos cuyos directores informan que sus centros hacen públicos sus datos de evaluaciones son en la media de países de la OCDE del $45 \%$, y en el caso de España tan solo son del $12.8 \%$. Estos datos afirman la apuesta hecha por la evaluación de la LOMCE, como herramienta clave para la mejora del sistema educativo español.

En las prácticas de los directores de centros en torno a la evaluación, es de destacar como PISA recoge datos específicos sobre los usos que se dan a los resultados de las evaluaciones, y arroja luz sobre el liderazgo fuera del aula. Destaca el porcentaje de alumnos cuyo director informó que tiene como práctica habitual el uso de los resultados de la evaluación de los alumnos de 15 años para comparar su centro con el rendimiento de la región o a nivel nacional, siendo para la media de países de la OCDE 
del $63 \%$ y en España del $44 \%$; o los que la usan para comparar su centro con otros centros, que es para la media de países de la OCDE del 53\% y en España del 37\%. Estos datos apuntan a la necesidad de analizar con más detalle la influencia en el rendimiento académico de estas prácticas de dirección para la realidad española.

Los directores de los centros no solo usan la evaluación de los alumnos como práctica para medir y mejorar la calidad de la formación que ofrecen. PISA presenta prácticas concretas, en las que los directores en España están en la media de sus homólogos de países de la OCDE, o ligeramente por encima: la especificación por escrito del currículo y objetivos educativos del centro; especificación escrita de los estándares de rendimiento de los alumnos; recogida sistemática de los datos de asistencia, graduación y exámenes, desarrollo profesional de profesores; autoevaluaciones; evaluaciones externas; e información de los alumnos sobre las clases, profesores y recursos.

Sin embargo, en las prácticas que se enumeran a continuación, los directores españoles se encuentran considerablemente por debajo de sus homólogos de países de la OCDE: como mentores de profesores, donde el porcentaje de alumnos cuyos directores informaron que se realizaban dicha práctica fue del $72 \%$ de media en países de la OCDE y $26 \%$ de directores en España; consultar de manera regular con expertos para mejorar el centro, con $43 \%$ de directores de media en países de la OCDE y $27 \%$ en España; y la implementación de políticas estandarizadas, como un currículo de matemáticas del centro con materiales de instrucción compartidos y formación del profesorado acorde, con un $62 \%$ de directores en la media de países de la OCDE, y 38\% en España.

Además, PISA incide en la forma en la que se mide la calidad de la enseñanza impartida por los profesores en las aulas. La observación de clases aparece como una práctica con un porcentaje de alumnos en centros cuyo director informa que la llevan a cabo un $69 \%$ de directores de centros o similares, mientras que en España, el porcentaje es tan solo de un $10 \%$.

Como consecuencia de la evaluación de los profesores, los directores informaron de una serie de acciones en las que ellos estaban más o menos involucrados, dependiendo de la autonomía que el centro tenga sobre las mismas. Son cambio en el salario, algún tipo de recompensa monetaria, actividades de desarrollo profesional, cambios en la probabilidad de mejora profesional, reconocimiento público desde la dirección, cambios en las responsabilidades profesionales para hacer el trabajo más atractivo, y un papel en las iniciativas de desarrollo del centro. Para todas estas acciones, los centros españoles participantes en PISA estaban por debajo de la media de centros de la OCDE. Todas ellas están en directa relación con el grado de autonomía que los directores tienen en el control de los recursos humanos del centro.

Entre los elementos sobre los que las acciones del director tienen una influencia indirecta clara, PISA cita en primer lugar las ausencias y el llegar tarde por parte de los alumnos, mostrando cómo aquellos centros que tienen altos porcentajes de alumnos de estas características tienden a obtener peores resultados académicos, con una variación en aumento desde las primeras evaluaciones PISA, hasta llegar a un 16\%. Otro análisis 
del mismo aspecto arroja datos sobre que los centros con mayor autonomía para determinar el currículo y las políticas de evaluación tienden a ser aquellos con menos alumnos con ausencias. Lo mismo sucede con el ambiente disciplinario del centro, donde los centros con mayor rendimiento académico tienen un ambiente de disciplina más positivo. Estos dos factores y su interrelación, tienen que ser tratados a nivel de centro para ver el grado de causalidad entre ellos y el papel de la dirección en su evolución, y si apuntan a una tendencia en el comportamiento y acciones tomadas por los directores en cada caso. Así lo apunta el informe, haciendo énfasis en la importancia de atraer a los mejores profesionales a los centros más difíciles.

Como conclusión, se puede decir que PISA se posiciona en línea con las tendencias internacionales que presentan un perfil de directores eficaces que buscan un equilibrio entre liderazgo administrativo e instructivo, tal y como se confirma después de realizado el análisis de todas las prácticas arriba recogidas. Por otra parte, al comparar éstas con el nuevo catálogo de competencias de dirección de la LOMCE, se aprecia que las presentadas en la nueva ley distan todavía de estar cerca de dicho equilibrio, aunque se apuesta por ejercer mayor liderazgo en esas dos áreas.

\section{Los directores en TALIS}

La OCDE lanzó por primera vez en 2008 su Estudio Internacional sobre la Enseñanza y el Aprendizaje (TALIS) con la intención de recoger más información sobre los profesores, el entorno escolar, la enseñanza, y el impacto que estos tienen sobre el aprendizaje de los alumnos. Para ello, 24 países participantes encuestaron a sus profesores y directores de centros de secundaria obligatoria para recoger dicha información, y donde uno de los temas de investigación fue el papel y la función del liderazgo escolar. Posteriormente, en el año 2013 se llevó a cabo la segunda ronda de TALIS, ahora con 34 países participantes, cuyo marco conceptual era establecer indicadores internacionales sobre los profesores y la enseñanza con el objeto de ayudar a los países a revisar y desarrollar políticas para conseguir un proceso de enseñanza aprendizaje más efectivo (OCDE, 2014) La información que proporciona sobre los profesores, directores y los centros donde trabajan permite complementar datos y políticas que no han sido tratados previamente por otros informes como PISA.

El informe abre reconociendo el papel fundamental que el liderazgo de los centros juega al fomentar un entorno de enseñanza y aprendizaje efectivo, y expresa la intención de describir el papel de los líderes de los centros. Incide en que los directores tienen un conjunto de responsabilidades muy exigente y amplio. Manifiestan en general que se enfrentan a demandas en ocasiones incompatibles, y que encuentran dificultades ante la variedad y el número de las mismas. Todo ello requiere que los directores sean capaces de dirigir los recursos humanos y materiales, comunicarse con personas de diversos estamentos, tomar decisiones basadas en datos, y proporcionar el liderazgo instructivo que los profesores necesitan para ayudar a sus alumnos en la escuela. No es de sorprender que en la mayoría de los países participantes, la exigencia y responsabilidades de los directores son cada vez mayores: mayor autonomía conlleva en general un aumento en la autoridad de los directores a la hora de tomar decisiones, 
lo que a su vez se traduce en una mayor exigencia en los resultados obtenidos por medio de sus acciones. Todo ello va en línea con lo que articula la LOMCE al promover medidas destinadas a fomentar la calidad de los centros docentes.

Resalta TALIS que la dirección de centros es una prioridad para muchos países que están preocupados en mejorar el rendimiento de sus alumnos. Hace referencia a la literatura internacional sobre el tema, recordando que la contribución de los directores a la mejora de los alumnos es indirecta a través de su impacto en la escuela, su organización y clima, y en especial en los profesores y en lo que enseñan, y que dicha influencia no es claramente demostrable en todos los casos. Como probable razón de la dificultad para explicar dicha relación es - como esgrimen Marzano, Waters y McNulty (tal y como se cita en OCDE, 2014) - que el papel y funciones del director no están claramente definidos, al no existir una línea de investigación al respecto.

Como parte del proceso de recogida de datos para el estudio TALIS, se preguntó a los directores encuestados cómo distribuían su tiempo de trabajo en las escuelas. Los directores dijeron que, de media, dedican a las actividades a continuación detalladas la siguiente proporción de su tiempo (entre paréntesis se especifica la proporción de tiempo que los directores españoles dedican a las mismas): un $41 \%$ (36\%) de su tiempo dedicados a tareas administrativas o de liderazgo; $21 \%$ (24\%) a tareas relacionadas con el currículo y la enseñanza; $15 \%$ (15\%) a interacciones con los alumnos; $11 \%(13 \%)$ a interacciones con los padres o tutores; y $7 \%(6 \%)$ a contactos con la comunidad, negocios e industrias.

Posteriormente, pasa a enumerar las actividades que componen las tareas administrativas o de liderazgo, que tal y como las presenta TALIS, incluyen: tratar asuntos de recursos humanos o de personal; normativa; realización de informes; presupuesto del centro; preparación de horarios y organización de las clases, planificación estratégica; responder a peticiones de las autoridades locales, regionales o nacionales; y actividades de liderazgo y dirección.

A su vez, las actividades de liderazgo y dirección las desglosa en una serie de actividades a las que los directores dedican a menudo o muy a menudo tiempo, cuantificándolas de media en (entre paréntesis se presenta aquí los porcentajes equivalentes a las respuestas de los directores españoles): $75 \%(69 \%)$ a tomar medidas para asegurar que los profesores se sientan responsables de los resultados académicos de sus alumnos; $68 \%(56 \%)$ a tomar medidas para que los profesores se hagan responsables para mejorar sus habilidades para enseñar; $68 \%(93 \%)$ a colaborar con los profesores para solucionar problemas de disciplina en las clases; $66 \%(83 \%)$ a dar a los padres o tutores información relacionada con el centro y el rendimiento de los alumnos; $64 \%(59 \%)$ a tomar medidas para apoyar la cooperación entre profesores para desarrollar nuevas prácticas de enseñanza; $62 \%(45 \%)$ a colaborar con directores de otros centros; $61 \%(65 \%)$ a comprobar errores en procedimientos e informes administrativos del centro; $49 \%(29 \%)$ a observar la instrucción en el aula; y $47 \%$ $(52 \%)$ a resolver problemas con los horarios de las clases. 
Las tareas relacionadas con el currículo y la enseñanza las concreta en: desarrollar el currículo, enseñar, observación de clases, evaluación de alumnos, ser mentor de profesores, y desarrollo profesional de los profesores.

Además de estas series de tareas y actividades relacionadas entre ellas, TALIS informa aparte del porcentaje de directores que dedican tiempo a dos actividades relacionadas con un plan de desarrollo del centro, cuantificándolas de media en (entre paréntesis se presenta aquí los porcentajes equivalentes a las respuestas de los directores españoles): $89 \%$ (90\%) a uso del rendimiento y resultados de evaluación de los alumnos para desarrollar los objetivos y programas educativos del centro; y $79 \%$ $(40 \%)$ a creación de un plan de desarrollo profesional para el centro.

En relación al grado de autonomía de los centros, TALIS presenta una nueva relación de tareas, especificando el porcentaje de profesores cuyos directores indicaron que ejercen una considerable responsabilidad a nivel de centro, cuantificándolas de media en (entre paréntesis se presenta aquí los porcentajes equivalentes a las respuestas de los directores españoles): $75 \%$ (27\%) a nombrar y contratar profesores; $68 \%(26 \%)$ a despedir o suspender de empleo a profesores; 36\% (5\%) a establecer los salarios iniciales y tablas salariales de los profesores; $37 \%(5 \%)$ a determinar los aumentos salariales de los profesores; $82 \%$ (54\%) a decidir la asignación del presupuesto dentro del centro; $96 \%(93 \%)$ a establecer políticas y procedimientos disciplinarios a los alumnos; $79 \%$ (37\%) a establecer políticas de evaluación de alumnos, incluyendo evaluaciones regionales y nacionales; $81 \%(53 \%)$ a aprobar la admisión de alumnos al centro; 94\% (98\%) a decidir los materiales de aprendizaje del centro; 64\% (32\%) a determinar el contenido de los cursos, incluyendo los currículos regionales y nacionales; y 78\% (39\%) a decidir qué cursos se ofertan.

Posteriormente, y sobre la misma serie de tareas, TALIS analiza el grado de responsabilidad compartida de los directores, y sobre todas las tareas arriba expuestas, a excepción de establecer políticas y procedimientos disciplinarios a los alumnos cuyo porcentaje está a la par - en el resto de tareas los directores españoles se encuentran significativamente por debajo en relación al nivel de distribución de responsabilidades de la media de los directores del estudio.

Con todos estos datos, TALIS plantea la construcción de un perfil del rol de director que se puede usar para apoyar al desarrollo de estándares para la profesión. Hay que considerar la gran variación existente en la participación de los directores en actividades administrativas y de liderazgo. No es el caso a la hora de diseñar los objetivos educativos del centro, donde la mayoría de los directores están implicados. Las competencias en la LOMCE relacionadas con esta dimensión se aproximan al número de prácticas que recoge TALIS al analizarlas de manera comparativa.

Finalmente, se destaca que TALIS hace referencia al liderazgo instructivo de los directores, diciendo que se encuentra presente en la mayoría de las acciones que realizan, incluyendo el asegurarse que los objetivos de la escuela están definidos, que el ambiente escolar es seguro y conducente al aprendizaje, y que el esfuerzo de los profesores está centrado en la enseñanza y en su mejora a la hora de enseñar, interpretándolo en las tareas de fijar los objetivos y programas, planificar el desarrollo 
profesional, y las tareas relacionadas con el currículo y la enseñanza. Relacionado con esta afirmación, indica que los directores que muestran un alto grado de liderazgo instructivo dedican más tiempo a tareas relacionadas con la enseñanza, el aprendizaje y el desarrollo de las prácticas de los profesores.

Debido a esto, las cualidades personales y las habilidades sociales e interpersonales son una de las áreas más importantes que definen la práctica de los directores, que deben ser complementadas con visión y valores, además del conocimiento y la comprensión, llevándolas a cabo al liderar la enseñanza y el aprendizaje, el desarrollo propio y de los demás, mejorar e innovar, dirigir el centro, e implicarse y trabajar con la comunidad.

Analizadas sus prácticas en conjunto, se puede afirmar que TALIS está en línea con el objetivo apuntado desde la literatura internacional de buscar un equilibrio entre liderazgo instructivo y administrativo - tal y como se veía con PISA. Si éstas se comparan con las competencias LOMCE, se aprecia una semejanza en el tratamiento de las prácticas asociadas con el liderazgo administrativo, mientras que las relacionadas con el liderazgo instructivo aún difieren en buena medida.

\section{¿Qué liderazgo se demanda desde los estudios internacionales?}

Después de la revisión comparativa de los datos aportados por PISA y TALIS relacionados con las prácticas de los directores y la función de dirección con las competencias de dirección que plantea la LOMCE, se presentan los siguientes resultados:

Se puede ratificar el empeño de la LOMCE en basar su desarrollo y filosofía de cambio en la información y datos empíricos presentados por los informes internacionales en lo que se refiere a la dirección de centros, puesto que conecta como pilares de su reforma - con una serie de cuestiones que dichos informes enfatizan recurrentemente, y que no son otros más que la necesidad de brindar mayor autonomía a los centros, unido a una rendición de cuentas por las acciones realizadas, tal y como se ha mostrado en el apartado anterior. Sobre la figura del director, se pretende que ejerza un liderazgo más pedagógico y de gestión, en sintonía con lo que la OCDE ya aconsejaba para los directores en su informe Mejorar el liderazgo escolar (Pont et al, 2008). Los informes internacionales presentan mayor equilibrio entre los dos tipos de liderazgo, mientras que en el caso de la LOMCE, se encuentra aún a camino de lograrlo, predominando las prácticas de gestión. Como se ve en TALIS, el porcentaje de tiempo dedicado a tareas instructivas y administrativas varía de unos países a otros, desde los extremos de Holanda (18\% y 54\% respectivamente) y Chile $(27 \%$ y $30 \%)$, pasando por España (24\% y $36 \%)$.

Ambos informes y la LOMCE utilizan diferentes términos para referirse a lo que hacen los directores. Hablan de acciones, tareas, competencias, prácticas o actividades a las que dedican el tiempo los directores. Los términos se utilizan indistintamente, sin concretar la diferencia en el uso de unos u otros, y sin haber sido definidos en su significado y límites. Dentro de la diversidad presentada, el número de prácticas de 
dirección en total recogidas en cada informe varía: PISA tiene un número ligeramente superior a TALIS, mientras que el encontrado en la LOMCE es sensiblemente inferior.

A efectos comparativos, se han clasificado las diferentes prácticas de cada informe junto con las competencias LOMCE, agrupándolas en torno a las cuatro dimensiones de la función de dirección que propone el informe Mejorar el Liderazgo Escolar (2008). Se aprecia cómo dichas dimensiones no se encuentran claramente reflejadas en las agrupaciones propuestas por PISA, TALIS, o competencias LOMCE. En el caso de PISA, las prácticas de dirección se agrupan en torno a índices: de responsabilidad escolar para la asignación de recursos y para el currículo y la evaluación; y de dirección escolar sobre la participación del profesorado, delimitar y comunicar los objetivos del centro y el desarrollo curricular, liderazgo instructivo, y promover la mejora en la instrucción y el desarrollo profesional. En el caso de TALIS, las prácticas de dirección se agrupan en torno a tareas administrativas o de liderazgo; relacionadas con el currículo y la enseñanza; con las interacciones con los alumnos, con los padres, y con la comunidad, negocios e industrias. En el caso de la LOMCE, las competencias del director no plantean ninguna agrupación.

En cuanto al número de prácticas que contiene cada dimensión, no existe uniformidad. En ocasiones, dentro de una dimensión, la misma práctica aparece en los dos informes y en la LOMCE. En otras, sin embargo, hay prácticas que cita un informe que no aparecen en los otros dos para la misma dimensión. Incluso sucede que algunas prácticas están repetidas para diferentes dimensiones desde el mismo informe.

Como resumen de la diversidad expuesta, se aprecia una descompensación en el número de prácticas que cada informe y la LOMCE tienen por dimensión, al igual que unas dimensiones tienen más preponderancia que otras entre informes. Respecto a las competencias del director propuestas por la LOMCE, la mayoría se agrupan en torno a la dimensión administrativa y de relaciones fuera del aula, mientras que dimensiones como la instructiva o de evaluación apenas recogen alguna competencia.

Esta es la tendencia que se describe sobre la función de dirección en España desde los informes internacionales, pero que en definitiva se corresponde con la dimensión de liderazgo a la que los directores dedican más tiempo, según se ha demostrado también por dichos informes.

\section{Hacia una mejora en la eficacia del liderazgo directivo}

Los dos informes internacionales aquí revisados subrayan la necesidad de un aumento de la autonomía en la dirección de los centros que ha de ir seguida de una rendición de cuentas sobre los objetivos académicos de los alumnos. Inciden en lo estático que ha permanecido el sistema educativo español a lo largo de los últimos años, indicando la necesidad de cambio para mejorar sus resultados. Entre sus conclusiones, este artículo suscribe el objetivo de la LOMCE de dar respuesta a dichas sugerencias, y proponer la evidencia empírica que parta de la investigación internacional como motor de cambio educativo, en este caso, sobre la figura de la dirección escolar 
También se ha probado cómo diversos estudios e informes internacionales no muestran acuerdo en el conjunto de prácticas que desempeñan los directores en su trabajo diario, ni en las dimensiones en las que dichas prácticas se agrupan. Se insiste en la idoneidad de dedicar más tiempo a prácticas parte de liderazgos instructivos y distributivos, reduciendo aquellas puramente burocráticas, a pesar de que la realidad muestra que dedican un tiempo considerable a estas últimas. Lo heterogéneo de las propuestas lleva a estudiar las prácticas de los directores no como parte de tipos de liderazgo concretos, sino más bien con la intención de construir una lista de actividades que definan el trabajo real de los directores y ver el grado de efectividad a la hora de realizarlas.

Esta labor apunta en tres direcciones: en primer lugar, al estudio detallado de las acciones de los directores proporcionadas por los informes internacionales aquí revisados, en base a la efectividad de sus prácticas; en segundo lugar, a la realización de una revisión de la literatura académica reciente en torno a las prácticas de los directores para conducir el mismo proceso comparativo y de síntesis entre las propuestas presentadas; y en tercer lugar, mirar a las políticas educativas en torno al liderazgo de centros que otros países han desarrollado, para recoger las contribuciones hechas en relación a la ordenación de esas prácticas, y sus consecuencias que tengan aplicación en la realidad española.

Con la información del paso anterior, se plantea el análisis detallado de las competencias prescritas en la LOMCE, y haciendo uso de los datos que PISA y TALIS proporcionan, estudiar su adecuación a la práctica habitual de los directores, probando la efectividad de dichas competencias a la luz del rendimiento académico. Con ello se podrá obtener un conjunto de prácticas o competencias de los directores que demuestren su validez ante la evidencia, y que permitan el desarrollo de la autonomía de centros de manera eficaz.

Si como se ha visto, la autonomía de los centros educativos va acompañada de rendición de cuentas de los diversos elementos que los componen, la evaluación de los directores en concreto debe ir precedida de la definición precisa de sus funciones en base a las actividades que desarrollan, para poder así medir su efectividad. Para poder evaluar de manera eficaz, se ha de hacer con relación a unos criterios fácilmente reconocibles que sirven de referente. En el caso de los directores, vienen dados en muchos casos por el diseño de unos estándares que se utilizan como herramientas de medida, que apuntan a acciones realizadas habitualmente por los directores, y que son fácilmente observables y reconocibles como reales por estos. La tradición en el desarrollo de estándares está ya consolidada en países como Inglaterra, Estados Unidos, Nueva Zelanda, Canadá entre otros, tal y como muestra un informe del CEPPE (2013).

Los estándares describen los niveles deseables de rendimiento a alcanzar para cada una de las acciones descritas, sobre las cuales los directores son medidos en su práctica. Los estándares deben ser construidos en base a la investigación empírica que analice las prácticas de los directores y la teoría sobre liderazgo educativo, y de esta manera proporcionarán una visión fiel del perfil de dirección más eficaz. Este es el 
camino que señala este artículo, siguiendo la propuesta de reforma hecha desde la nueva ley educativa.

\section{Referencias bibliográficas}

BARBER, M., \& MOURSHED, M. (2007). How the world's best-performing school systems come out on top. London: McKinsey \& Company.

CARD, N. (2012). Applied meta-analysis for social science research. New York: The Guilford Press.

CUBAN, L. (1988). The managerial imperative and the practice of leadership in schools. Albany, NY: State University of New York Press.

FULLAN, M. (2002). The change leader. Educational Leadership, 59(8), 15-20.

GRISSOM, J. A., \& LOEB, S. (2011). Triangulating principal effectiveness: How perspectives of parents, teachers, and assistant principals identify the central importance of managerial skills. American Education Research Journal, 48(5), 1091-1123.

HALLINGER, P., \& HECK, R. (1998). Exploring the principal's contribution to school effectiveness: 1980-1995. School Effectiveness and School Improvement, 9(2), 157-191.

INSTITUTO NACIONAL DE EVALUACIÓN EDUCATIVA (2013). PISA 2012. Informe español. Madrid: Ministerio de Educación, Cultura y Deporte.

LEITHWOOD, K., DAY, C., SAMMONS, P., HARRIS, A., \& HOPKINS, D. (2006). Successful school leadership: What it is and how it influences pupil learning. Nottingham, UK: National College for School Leadership and Department for Education and Skills.

LEITHWOOD, K., HARRIS, A., \& HOPKINS, D. (2008). Seven strong claims about successful school leadership. School Leadership and Management, 28(1), 27-42.

MULFORD, B. (2003). School leaders: Changing roles and impact on teacher and school effectiveness. Paris: OECD Publishing.

SEASHORE-LOUIS, K., LEITHWOOD, K., WAHLSTROM, K., \& ANDERSON, S. (2010). Investigating the links to improved student learning: Final report of research findings. Minneapolis, MN: University of Minnesota, Center for Applied Research and Educational Improvement; Toronto, ON: University of Toronto, OISE.

WATERS, T., MARZANO, R., \& MCNULTY, B. (2005). Balanced leadership: What 30 years of research tells us about the effect of leadership on student achievement. Denver, CO: Mid-continent Research for Education and Learning.

YUKL, G.A. (2002). Leadership in organizations $\left(5^{\text {th }}\right.$ ed). Upper Saddle River, NJ: Prentice Hall. 


\section{Referencias digitales}

CEPPE (2013). Learning standards, teaching standards and standards for school principals: A comparative study. OECD Education Working Papers, 99. Paris: OECD Publishing. Recuperado de http://dx.doi.org/10.1787/5k3tsjqtp90v-en (Consultado 4 enero de 2015).

COMISIÓN EUROPEA/EACEA/Eurydice (2013). Cifras clave del profesorado y la dirección de centros educativos en Europa. Edición 2013. Informe de Eurydice. Luxemburgo: Oficina de Publicaciones de la Unión Europea. Recuperado de http://eacea.ec.europa.eu/education/eurydice./documents/key_data_series/151ES.pd f (Consultado 1 de marzo de 2015).

INGVARSON, L. C., ANDERSON, M., GRONN, P., \& JACKSON, A. (2006). Standards for school leadership: A critical review of the literature. Canberra: Teaching Australia. Recuperado de http://www.aitsl.edu.au/docs/defaultsource/default-document-library/standards_for_school_leadership__a_critical_review_of_literature (Consultado $\overline{6}$ de diciembre de 2013 ).

LEY ORGÁNICA 2/2006, de 3 de mayo, de Educación. Boletín Oficial del Estado (España), 4 de mayo de 2006, 106, 17158-17207. Recuperado de http://www.boe.es/boe/dias/2006/05/04/pdfs/A17158-17207.pdf_(Consultado 12 de diciembre de 2013).

LEY ORGÁNICA 8/2013, de 9 de diciembre, de Mejora de la Calidad Educativa. Boletín Oficial del Estado (España), 10 de diciembre de 2013, 295, 97858-97921. Recuperado de http://www.boe.es/boe/dias/2013/12/10/pdfs/BOE-A-201312886.pdf (Consultado 12 de junio de 2014).

OCDE (2013). PISA 2012 results: What makes a school successful (volume IV): Resources, policies and practice. Paris: OECD Publishing. doi: 10.1787/9789264201156-en_(Consultado 28 de junio de 2014).

OCDE (2014). TALIS 2013 results: An international perspective on teaching and learning. Paris: OECD Publishing. doi: 10.1787/9789264196261-en_(Consultado 19 de agosto de 2014).

PONT, B., NUSCHE, D., \& MOORMAN, H. (2008). Improving school leadership, volume 1: Policy and practice. Paris: OECD Publishing. doi: 10.1787/9789264044715-en_(Consultado 15 de enero de 2014).

WITZIERS, B., BOSKER, R., \& KRÜGER, M. (2003). Educational leadership and student achievement: The elusive search for an association. Educational Administration Quarterly, 39(3), 398-425. doi: 10.1177/0013161X03253411 (Consultado 18 de noviembre de 2013). 


\section{Correspondencia con el autor}

\section{David IZQUIERDO GÓMEZ}

IES Julio Verne

$\mathrm{C} /$ Ingeniería, $\mathrm{s} / \mathrm{n}$

28918, Leganés, Madrid.

e-mail: dizquierdogomez@educa.madrid.org 